\title{
Anaphylactic Reaction in a Patient Undergoing Open Appendicectomy and Right Hemicolectomy
}

\author{
Tavares F, Ferraz I, Castro P. Pires AC
}

\section{INTRODUCTION}

Latex is one of the most common causes of intraoperative anaphylactic reactions. ${ }^{1}$ Despite the increasing number of detected latex allergies in general population, severe anaphylactic reactions during surgical procedures are still rare.

\section{Case Report:}

> A 19-year-old male presented for an emergency appendicectomy and right hemicolectomy after a car accident.

$>$ No allergies were reported, and his preoperative medical history and physical examination were unremarkable.

$>$ A rapid sequence induction was performed with fentanyl, propofol and rocuronium, and anaesthesia maintained with desflurane.

> 50 minutes later, patient presented bronchospasm, tachycardia (135 beat $/ \mathrm{min})$, hypotension $(68 / 40 \mathrm{mmHg})$ and desaturation $\left(\mathrm{SpO}_{2} 89 \%\right)$. Wheezing and rales were detected. Ventilation with $100 \% \mathrm{O}_{2}$ was administered and desflurane was replaced by sevoflurane. One more large bore access were placed and rapid infusion of $1500 \mathrm{~mL}$ of Ringer's Lactate was initiated.

> Drugs administered: Phenylephrine 100 mg, hydrocortisone $200 \mathrm{mg}$ and 6 "puffs" of salbutamol.

\section{The patient's vital signs were recovered}

> At the end of the surgery, patient presented once again bronchospasm, tachycardia and desaturation. Skin eruptions involving the whole body and periorbital angioedema were observed.

> Posteriorly administered: adrenalin $5 \mu \mathrm{g} / \mathrm{kg}(350 \mu \mathrm{g})$ and repeated salbutamol.

$>10$ minutes later, after vital signs returned to normal, the patient was intubated and ventilated transferred to the ICU. He remained hemodynamically stable during the postoperative course and was discharged 3 days later.

$>$ Posteriorly, family referred that the patient used to present periorbital and perioral edema when blowing rubber balloons during his childhood.

\section{We assumed an anaphylactic reaction to latex}

\section{Discussion}

Anaphylaxis is a life-threatening, unpredictable, and dose independent reaction. An early recognition of signs and symptoms is essential for immediate treatment and prevention of adverse outcomes. A high suspicion index is also extremely important.

\section{Learning points}

After clinical stabilization, measures should be taken to confirm diagnostic hypothesis (levels of tryptase). These patients' follow-up is extremely important in order to provide safer future procedures. 